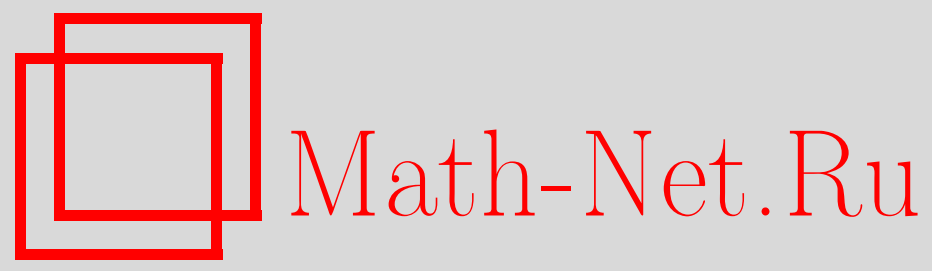

А. В. Еремин, Н. М. Будыльников, И. В. Кудинов, Теплообмен при течении Куэтта с учётом диссипации энергии при граничных условиях третьего рода, Вестн. Сам. гос. техн. ун-та. Сер. Физ.-мат. науки, 2012, выпуск 3(), 136-144

DOI: https://doi.org/10.14498/vsgtu1065

Использование Общероссийского математического портала Math-Net.Ru подразумевает, что вы прочитали и согласны с пользовательским соглашением

http: //www . mathnet.ru/rus/agreement

Параметры загрузки:

IP : 54.198 .67 .100

26 апреля 2023 г., 10:01:11

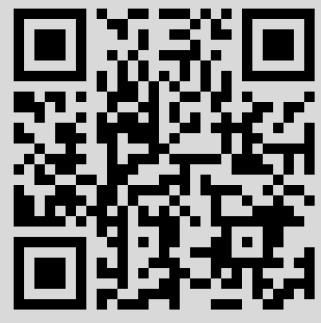




\title{
ТЕПЛООБМЕН ПРИ ТЕЧЕНИИ КУЭТТА С УЧЁТОМ ДИССИПАЦИИ ЭНЕРГИИ ПРИ ГРАНИЧНЫХ УСЛОВИЯХ ТРЕТЬЕГО РОДА
}

\author{
А. В. Еремин ${ }^{1}$, Н. М. Будъльников ${ }^{1}$, И. В. Кудинов ${ }^{2}$ \\ 1 Самарский государственный технический университет, \\ 443100, Россия, Самара, ул. Молодогвардейская, 244. \\ 2 Самарский государственный архитектурно-строительный университет, \\ 443001, Россия, Самара, ул. Молодогвардейская, 194 \\ E-mails: a.v.eremin@list.ru, budylnikov@mail.ru, igor_koudinov@mail.ru
}

\begin{abstract}
Путём совместного использования метода Л. В. Канторовича и ортогонального метода Бубнова-Галёркина получено аналитическое решение нелинейной задачи теплообмена при ламинарном течении жидкости в плоскопараллельном канале (течение Куэтта) с учётом диссипации энергии при задании граничных условий третъего рода на движущейся стенке. Решение позволяет выполнять оценку температурного состояния жидкости при малых значениях продолъной координать, где, как показали исследования, происходит смещение профиля температуры в сторону неподвижной стенки.
\end{abstract}

Ключевые слова: течение Куэтта, теплообмен в жидкости, аналитическое решение, ортогональные методь, диссипация энергии.

Проведем анализ теплообмена в жидкости, находящейся между двумя плоскими стенками, одна из которых движется относительно другой с некоторой постоянной скоростью $\omega_{0}$ (теплообмен при течении Куэтта [1]). Профиль скорости такого течения описывается линейной функцией от поперечной координаты $y$. Аналогичный профиль скорости формируется в ламинарном вязком подслое турбулентного пограничного слоя. Излучение процессов теплообмена в пограничном слое вязкой жидкости с учётом теплоты трения (диссипация энергии) представляет значительный интерес в технике. При высоких скоростях течения среды (или высокоскоростном движении технических аппаратов в среде) повышение температуры от диссипации энергии может значительно превосходить изменение температуры от всех других источников теплоты. При этом особый интерес представляют задачи, когда со стороны движущейся пластины (аппарата) теплообмен происходит при граничных условиях третьего рода (теплопроводностью пластины пренебрегают).

Подобные задачи являются нелинейными - их точные аналитические решения в настоящее время не получены. Известные приближенные решения найдены лишь при граничных условиях первого рода на стенках [1]. В работе [1] путем совместного использования интегральных преобразований Лапласа и ортогонального метода Бубнова-Галёркина получено решение данной задачи лишь в третьем приближении, что не позволяет проводить исследование теплообмена для малых значений продольной координаты. Увеличение числа приближений по методу [1] связано с необходимостью решения в

Антон Владимирович Еремин, аспирант, каф. теоретических основ теплотехники и гидромеханики. Николай Михайлович Будыльников, аспирант, каф. теоретических основ теплотехники и гидромеханики. Игорь Василъевич Кудинов (к.т.н.), ассистент, каф. гидравлики и теплотехники. 
общем виде (в изображениях по Лапласу) систем алгебраических уравнений большой размерности, содержащих параметр интегрального преобразования. В данном случае относительно изображения искомой функции получаются столь сложные выражения, что обратный переход от изображения к оригиналам оказывается практически неосуществимым ввиду отсутствия соответствующих стандартных формул для выполнения такого перехода. В то же время отметим, что получение решения для начального участка продольной координаты связано с существенным увеличением числа приближений. $\mathrm{K}$ тому же исследование теплообмена именно на этом участке представляет наибольший интерес, особенно в случаях, когда мощность теплового потока от диссипации энергии становится соизмеримой со всеми другими видами переноса теплоты в пограничном слое (движение тел при больших скоростях) или превышает их.

В настоящей работе для решения указанной задачи применен ортогональный метод Л. В. Канторовича, с помощью которого получено решение в пятом приближении. Каких-либо принципиальных трудностей, связанных с дальнейшим увеличением числа приближений, не возникает. При этом уже в пятом приближении найдено решение для значений безразмерной продольной координаты $\eta=1 \cdot 10^{-4}$, что позволило получать результаты, отсутствующие в известной литературе.

Рассмотрим задачу Куэтта при граничных условиях третьего рода на движущейся стенке (рис. 1). Математическая постановка

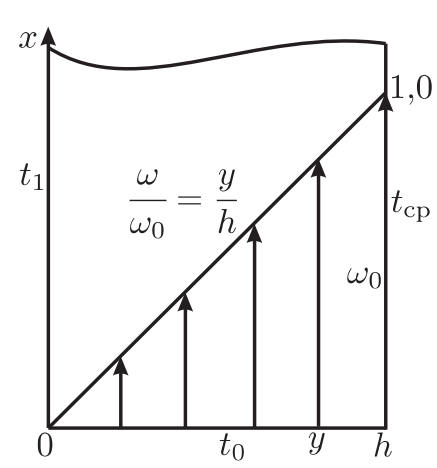

Рис. 1. Схема течения Куэтта при граничных условиях третьего рода на движущейся стенке задачи имеет вид

$$
\begin{gathered}
\omega(y) \frac{\partial t(x, y)}{\partial x}=a \frac{\partial^{2} t(x, y)}{\partial y^{2}}+\frac{\mu}{\rho}\left(\frac{d \omega}{d y}\right)^{2}, \quad 0 \leqslant y \leqslant h ; 0 \leqslant x<\infty ; \\
t(0, y)=t_{0} ; \\
t(x, 0)=t_{1} ; \\
\lambda \frac{\partial t(x, h)}{\partial y}+\alpha\left[t(x, h)-t_{\mathrm{cp}}\right]=0,
\end{gathered}
$$

где $\omega(y)=\omega_{0} y / h-$ профиль скорости плоскопараллельного течения (течение Куэтта); $h$ - ширина канала; $y, x$ - поперечная и продольная координаты; $\mu$-динамическая вязкость; $\rho$ - плотность; $a$-коэффициент температуропроводности; $\lambda$ - коэффициент теплопроводности; $t$ - температура; $t_{0}$ - температура жидкости на входе в канал $(x=0) ; t_{1}$ - температура невозмущенного потока (при $y=0), t_{\text {ср }}$ - температура среды, омывающей подвижную пластину при $y=h$ (толщиной пластины и ее теплопроводностью пренебрегают); $\omega_{0}$ - скорость подвижной пластины; $\alpha$ - коэффициент теплоотдачи.

Введём безразмерные переменные $\eta=a x /\left(\omega_{0} h^{2}\right), \xi=y / h$, в которых 
задача (1)-(4) запишется следующим образом:

$$
\begin{gathered}
\xi \frac{\partial t(\eta, \xi)}{\partial \eta}=\frac{\partial^{2} t(\eta, \xi)}{\partial \xi^{2}}+R, \quad 0 \leqslant \eta<\infty ; 0 \leqslant \xi \leqslant 1 \\
t(0, \xi)=t_{0} \\
t(\eta, 0)=t_{1} \\
\frac{\partial t(\eta, 1)}{\partial \xi}+\operatorname{Bi}\left[t(\eta, 1)-t_{\mathrm{cp}}\right]=0
\end{gathered}
$$

где $\mathrm{Bi}=\alpha h / \lambda-$ число Био, $R=\mu \omega_{0}^{2} / \lambda$. Для приведения граничных условий $(7),(8)$ к однородным введём новую функцию $T(\eta, \xi)$, связанную с функцией $t(\eta, \xi)$ зависимостью

$$
t(\eta, \xi)=T(\eta, \xi)+b_{1}+b_{2} \xi
$$

где $b_{1}, b_{2}$ - неизвестные коэффициенты. Задача (5)-(8) относительно функции $T(\eta, \xi)$ примет вид

$$
\begin{gathered}
\xi \frac{\partial T(\eta, \xi)}{\partial \eta}=\frac{\partial^{2} T(\eta, \xi)}{\partial \xi^{2}}+R \\
T(\eta, \xi)=t_{0}-b_{1}-b_{2} \xi \\
T(\eta, 0)=t_{1}-b_{1} \\
\frac{\partial T(\eta, 1)}{\partial \xi}+\operatorname{BiT}(\eta, 1)=-\operatorname{Bi} b_{1}-(1+\mathrm{Bi}) b_{2}+t_{\mathrm{cp}}
\end{gathered}
$$

Требование однородности граничных условий $(11),(12)$ приводит к системе уравнений, из решения которой находятся коэффициенты $b_{1}, b_{2}$ :

$$
b_{1}=t_{1} ; \quad b_{2}=\frac{\mathrm{Bi}\left(t_{\mathrm{cp}}-t_{1}\right)}{(1+\mathrm{Bi})} .
$$

C учётом найденных значений коэффициентов $b_{1}$ и $b_{2}$ задача (9)-(12) запишется так:

$$
\begin{gathered}
\xi \frac{\partial T(\eta, \xi)}{\partial \eta}=\frac{\partial^{2} T(\eta, \xi)}{\partial \xi^{2}}+R \\
T(0, \xi)=\Delta t-b_{2} \xi \\
T(\eta, 0)=0 \\
\frac{\partial T(\eta, 1)}{\partial \xi}+\operatorname{BiT}(\eta, 1)=0
\end{gathered}
$$

где $\Delta t=t_{0}-t_{1}$.

Решение задачи (13)-(16) принимается в виде

$$
T(\eta, \xi)=\sum_{k=1}^{n} f_{k}(\eta) \varphi_{k}(\xi),
$$


где $\varphi_{k}(\xi)=r \xi+\xi^{2 k}-$ координатные функции; $f_{k}(\eta)-$ неизвестные функции; $r$ - коэффициент, определяемый таким образом, чтобы выполнялось однородное граничное условие (16).

Подставляя (17) в (16), относительно $r$ получаем алгебраическое линейное уравнение, из решения которого находим $r=-(2 k+\mathrm{Bi}) /(1+\mathrm{Bi})$. С учётом найденного значения коэффициента $r$ соотношение $(17)$ в любом приближении удовлетворяет однородным граничным условиям (15), (16) при любых значениях $f_{k}(\eta)$.

Для нахождения решения (13)-(16) в первом приближении составим невязку уравнения (13) и потребуем ортогональности невязки к координатной функции $\varphi_{1}(\xi)=r \xi+\xi^{2}$, то есть используем метод Л. В. Канторовича [3]:

$$
\int_{0}^{1}\left[\xi \frac{\partial T(\eta, \xi)}{\partial \eta}-\frac{\partial^{2} T(\eta, \xi)}{\partial \xi^{2}}-R\right] \varphi_{1}(\xi) d \xi=0 .
$$

Подставляя (17) в (18) (ограничимся одним членом ряда), после вычисления интегралов относительно неизвестной функции $f_{1}(\eta)$ получаем обыкновенное дифференциальное уравнение

$$
\frac{d f_{1}}{f_{1}-0,5 R}=\left(\frac{2}{3}+r\right) \frac{d \eta}{\nu}
$$

из которого находим

$$
f_{1}(\eta)=C \exp (\nu \eta)-0,5 R
$$

Здесь $\nu=1 / 6+2 r / 5+r^{2} / 4, C-$ постоянная интегрирования. Подставляя (19) в (17), получим

$$
T(\eta, \xi)=[C \exp (\nu \eta)-0,5 R](r+\xi) \xi
$$

Постоянная интегрирования $C$ находится из условия ортогональности невязки начального условия (14) к координатной функции $\varphi_{1}(\xi)$, т. е.

$$
\int_{0}^{1}\left[(C \exp (\nu \eta)-0,5 R)(r+\xi) \xi-\Delta t+b_{2} \xi\right](r+\xi) \xi d \xi=0
$$

После вычисления интеграла в (21) определим

$$
C=\frac{R A+(0,33333+0,5 r) \Delta t-(0,25+0,33333 r)}{0,2+0,5 r+0,33333 r^{2}}
$$

где $A=0,1+0,16667 r^{2}+0,25 r$. Таким образом, решение задачи $(5)-(8)$ в первом приближении находится из (20).

Найдём решение для случая, когда температура зависит лишь от диссипации энергии, то есть при $t_{0}=t_{1}=t_{\text {ср }}$. Положим также, что $\mathrm{Bi} \rightarrow \infty$ и, следовательно, $r \rightarrow 1$. Соотношение (20) для безразмерной температуры $\Theta(\eta, \xi)=\left(t-t_{0}\right) / R$ приводится к виду

$$
\Theta(\eta, \xi)=0,5[1-\exp (-20 \eta)](1-\xi) \xi
$$

которое совпадает с решением этой задачи в первом приближении, полученным в $[1,2,4]$. 
Для нахождения решения во втором приближении составляется невязка уравнения (13) и требуется ортогональность невязки к координатным функциям $\varphi_{1}(\xi)$ и $\varphi_{2}(\xi)$ :

$$
\int_{0}^{1}\left[\xi\left(\frac{\partial f_{1}}{\partial \eta} \varphi_{1}+\frac{\partial f_{2}}{\partial \eta} \varphi_{2}\right)-f_{1} \frac{\partial^{2} \varphi_{1}}{\partial \xi^{2}}-f_{2} \frac{\partial^{2} \varphi_{2}}{\partial \xi^{2}}-R\right] \varphi_{j}(\xi) d \xi=0, j=1,2 .
$$

Вычисляя интегралы в (22), относительно неизвестных функций $f_{1}(\eta)$ и $f_{2}(\eta)$ получаем систему обыкновенных дифференциальных уравнений

$$
\left\{\begin{array}{l}
A_{11} f_{1}^{\prime}+A_{12} f_{2}^{\prime}+B_{11} f_{1}+B_{12} f_{2}+N_{1}=0 \\
A_{21} f_{1}^{\prime}+A_{22} f_{2}^{\prime}+B_{21} f_{1}+B_{22} f_{2}+N_{2}=0
\end{array}\right.
$$

где

$$
\begin{aligned}
& A_{11}=\int_{0}^{1} \xi \varphi_{1} \varphi_{1} d \xi=\frac{1}{60} ; \quad A_{12}=\int_{0}^{1} \xi \varphi_{1} \varphi_{2} d \xi=\frac{1}{105} \\
& B_{11}=-\int_{0}^{1} \xi \varphi_{1}^{\prime \prime} \varphi_{1} d \xi=-\frac{1}{3} ; \quad B_{12}=-\int_{0}^{1} \varphi_{2}^{\prime \prime} \varphi_{1} d \xi=-\frac{1}{6} \\
& A_{21}=\int_{0}^{1} \xi \varphi_{1} \varphi_{2} d \xi=\frac{1}{105} ; \quad A_{22}=-\int_{0}^{1} \xi \varphi_{2} \varphi_{2} d \xi=\frac{1}{168} \\
& B_{21}=-\int_{0}^{1} \varphi_{1}^{\prime \prime} \varphi_{2} d \xi=-\frac{1}{6} ; \quad B_{22}=-\int_{0}^{1} \varphi_{2}^{\prime \prime} \varphi_{2} d \xi=-\frac{2}{15} \\
& N_{1}=-R \int_{0}^{1} \varphi_{1} d \xi=-\frac{R}{6} ; \quad N_{2}=-R \int_{0}^{1} \varphi_{2} d \xi=-\frac{R}{12}
\end{aligned}
$$

Решение системы (23) ищется в виде

$$
f_{1}(\eta)=\bar{f}_{1}(\eta)+f_{1}^{*}(\eta) ; \quad f_{2}(\eta)=\bar{f}_{2}(\eta)+f_{2}^{*}(\eta)
$$

где $\bar{f}_{1}(\eta), \bar{f}_{2}(\eta)$ - частные решения неоднородной системы уравнений $(23)$; $f_{1}^{*}(\eta), f_{2}^{*}(\eta)$ - общие решения соответствующей однородной системы.

Общие решения однородной системы находятся в виде

$$
\begin{aligned}
& f_{1}^{*}(\eta)=D_{11} \exp \left(\mu_{1} \eta\right)+D_{21} \exp \left(\mu_{2} \eta\right) \\
& f_{2}^{*}(\eta)=D_{12} \exp \left(\mu_{1} \eta\right)+D_{22} \exp \left(\mu_{2} \eta\right)
\end{aligned}
$$

где $D_{k j}, \mu_{k}$ - некоторые (пока неизвестные) постоянные.

Подставляя $(25)$ в (23) и положив $N_{1}=N_{2}=0$, получаем систему однородных алгебраических уравнений

$$
\left\{\begin{array}{l}
D_{11}\left(A_{11} \mu+B_{11}\right)+D_{21}\left(A_{12} \mu+B_{12}\right)=0 \\
D_{12}\left(A_{21} \mu+B_{21}\right)+D_{22}\left(A_{22} \mu+B_{22}\right)=0
\end{array}\right.
$$

которая имеет нетривиальное решение, если её определитель равен нулю. Раскрывая определитель, относительно $\mu$ получаем алгебраическое уравнение

$$
\mu^{2} / 5880+13 \mu / 630+1 / 3=0
$$


из решения которого находим $\mu_{1}=-19,19, \mu_{2}=-102,15$. Если положить $D_{11}=D_{21}=1$, то $D_{12}=0,8418, D_{22}=-1,6983$, то

$$
\begin{aligned}
& f_{1}^{*}(\eta)=C_{1} \exp \left(\mu_{1} \eta\right)+C_{2} \exp \left(\mu_{2} \eta\right), \\
& f_{2}^{*}(\eta)=C_{1} D_{12} \exp \left(\mu_{1} \eta\right)+C_{2} D_{22} \exp \left(\mu_{2} \eta\right),
\end{aligned}
$$

где $C_{1}$ и $C_{2}$ в дальнейшем (см. ниже формулу (32)) будут находиться из граничного условия (14). Соотношения (26) представляют общие решения однородной системы уравнений (23).

Частные решения $\bar{f}_{1}$ и $\bar{f}_{2}$ неоднородной системы уравнений (13) ищутся в виде общих решений $(26)$ в предположении, что $C_{1}$ и $C_{2}-$ функции от переменной $\eta$ :

$$
\begin{aligned}
& \bar{f}_{1}(\eta)=C_{1}(\eta) \exp \left(\mu_{1} \eta\right)+C_{2}(\eta) \exp \left(\mu_{2} \eta\right) \\
& \bar{f}_{2}(\eta)=C_{1}(\eta) D_{12} \exp \left(\mu_{1} \eta\right)+C_{2}(\eta) D_{22} \exp \left(\mu_{2} \eta\right)
\end{aligned}
$$

Подставляя (27) в (23), получим систему уравнений

$$
\left\{\begin{array}{l}
C_{1}^{\prime}(\eta) \nu_{1}\left(A_{11}+A_{12} D_{12}\right)+C_{2}^{\prime} \nu_{2}\left(A_{11}+A_{12} D_{12}\right)+N_{1}=0 \\
C_{1}^{\prime}(\eta) \nu_{1}\left(A_{21}+A_{22} D_{12}\right)+C_{2}^{\prime} \nu_{2}\left(A_{21}+A_{22} D_{12}\right)+N_{2}=0
\end{array}\right.
$$

где $\nu_{1}=\exp \left(\mu_{1} \eta\right), \nu_{2}=\exp \left(\mu_{2} \eta\right), C_{1}^{\prime}(\eta)=d C_{1} / d \eta, C_{2}^{\prime}(\eta)=d C_{2} / d \eta$, решение которой имеет вид

$$
\begin{gathered}
C_{1}(\eta)=-\frac{1}{\mu_{1} \nu_{1}} \frac{N_{1}\left(A_{21}+A_{22} D_{22}\right)-N_{2}\left(A_{11}+A_{12} D_{22}\right)}{\nu_{1}\left(D_{12}-D_{22}\right)\left(A_{11} A_{22}-A_{12} A_{21}\right)}+z_{1} ; \\
C_{2}(\eta)=\frac{1}{\mu_{2} \nu_{2}} \frac{N_{2}\left(A_{11}+A_{12} D_{12}\right)-N_{1}\left(A_{21}+A_{22} D_{12}\right)}{\nu_{2}\left(D_{12}-D_{22}\right)\left(A_{11} A_{22}-A_{12} A_{21}\right)}+z_{2} .
\end{gathered}
$$

Здесь $z_{1}$ и $z_{2}$ - постоянные интегрирования; так как находятся частные решения, можно принять $z_{1}=z_{2}=0$.

Подставляя (28) в (27), находим частные решения системы уравнений (23):

$$
\begin{aligned}
& \bar{f}_{1}(\eta)=\frac{1}{\mu_{1} \mu_{2} b_{5}}\left[\mu_{2}\left(N_{2} b_{1}-N_{1} b_{2}\right)+\mu_{1}\left(N_{1} b_{3}-N_{2} b_{4}\right)\right] \\
& \bar{f}_{2}(\eta)=\frac{1}{\mu_{1} \mu_{2} b_{5}}\left[\mu_{2}\left(N_{2} \delta_{1}-N_{1} \delta_{2}\right)+\mu_{1}\left(N_{1} \delta_{3}-N_{2} \delta_{4}\right)\right]
\end{aligned}
$$

где $\delta_{1}=D_{12}\left(A_{11}+A_{12} D_{22}\right) ; \delta_{2}=D_{12}\left(A_{21}+A_{22} D_{22}\right) ; \delta_{3}=D_{22}\left(A_{21}+A_{22} D_{12}\right)$; $\delta_{4}=D_{22}\left(A_{11}+A_{12} D_{12}\right) ; b_{1}=A_{11}+A_{12} D_{22} ; b_{2}=A_{21}+A_{22} D_{22} ; b_{3}=A_{21}+$ $+A_{22} D_{12} ; b_{4}=A_{11}+A_{12} D_{12} ; b_{5}=A_{11} A_{22}\left(D_{12}-D_{22}\right)+A_{12} A_{21}\left(D_{22}-D_{12}\right)$. Соотношения (29) удовлетворяют системе уравнений (23), в чём можно убедиться непосредственной подстановкой.

Подставляя (26), (29) в (24) получаем

$$
\begin{aligned}
& f_{1}(\eta)=C_{1} \exp \left(\mu_{1} \eta\right)+C_{2} \exp \left(\mu_{2} \eta\right)+\left(\mu_{2} \bar{r}_{1}+\mu_{1} \bar{r}_{2}\right) / r \\
& f_{2}(\eta)=C_{1} D_{12} \exp \left(\mu_{1} \eta\right)+{ }_{2} D_{22} \exp \left(\mu_{2} \eta\right)+\left(\mu_{2} r_{1}+\mu_{1} r_{2}\right) / r
\end{aligned}
$$

где $r=\mu_{1} \mu_{2} b_{5}, r_{1}=N_{2} \delta_{1}-N_{1} \delta_{2}, r_{2}=N_{1} \delta_{3}-N_{2} \delta_{4}, \bar{r}_{1}=N_{2} b_{1}-N_{1} b_{2}, \bar{r}_{2}=$ $=N_{1} b_{3}-N_{2} b_{4}$. Подставляя (30) в (17) (при Вi $\rightarrow \infty$ и $r=1$ ) будем иметь

$$
T(\eta, \xi)=f_{1}(\eta)(1-\xi) \xi+f_{2}(\eta)(1-\xi) \xi^{2} .
$$


Для определения постоянных $C_{1}$ и $C_{2}$ составим невязку граничного условия (14) и потребуем ортогональности невязки к координатным функциям $\varphi_{1}(\xi)$ и $\varphi_{2}(\xi)$ :

$$
\int_{0}^{1}\left[T(0, \xi)-\Delta t+b_{2} \xi\right] \varphi_{j}(\xi) d \xi=0, \quad j=1,2 .
$$

Подставляя (31) в (32), после вычисления интегралов получаем систему двух алгебраических линейных уравнений, из решения которой находим $C_{1}=-0,334267 R ; C_{2}=-0,165627 R$ (при учёте только диссипации энергии). Окончательные выражения для решения задачи (13)-(16) во втором приближении находятся в виде (30)-(31).

Результаты расчётов по формуле (31) в сравнении с [1] даны на рис. 2. Их анализ позволяет заключить, что для $\eta \geqslant 0,1$ результаты расчётов практически совпадают. Для $\eta=0,01$ расхождение не превышает $1 \%$, а для всех $\eta<0,01$ расхождение возрастает.

В случае $n$ приближений необходимо составлять невязку уравнения (13) и требовать ортогональности невязки к $n$ координатным функциям:

$$
\int_{0}^{1}\left[\sum_{k=1}^{n}\left(\xi f_{k}^{\prime} \varphi_{k}-f_{k} \varphi_{k}^{\prime \prime}\right)-R_{1}\right] \varphi_{j} d \xi=0, \quad j=1,2, \ldots, n .
$$

Вычисляя интегралы в $(33)$, относительно $f_{k}(\eta), k=1,2, \ldots, n$, придём к системе из $n$ обыкновенных дифференциальных уравнений. Последовательность дальнейшего получения решения аналогична рассмотренному выше процессу нахождения решения во втором приближении.

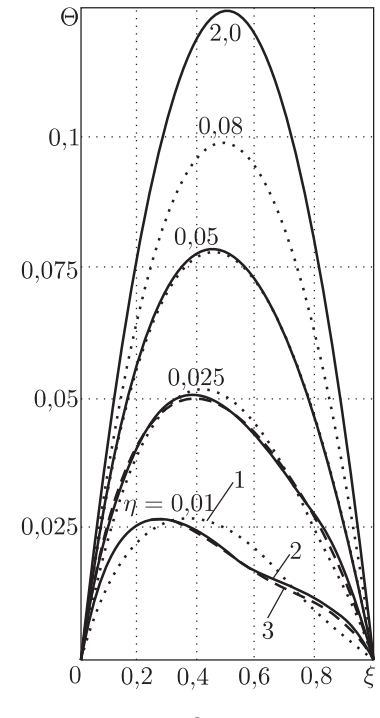

a

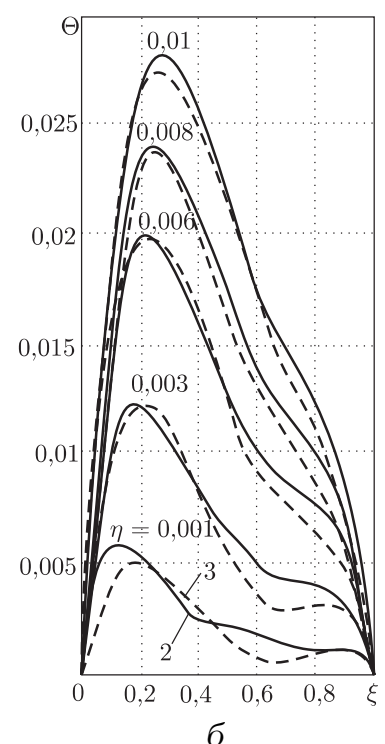

б

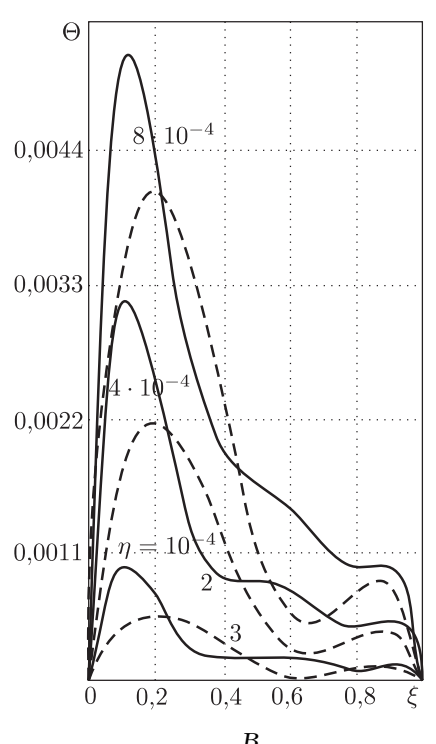

B

Рис. 2. Изменение температуры жидкости в плоском канале вследствие диссипации энергии: 1 - по формуле (17) при $n=2 ; 2$ - по формуле (17) при $n=5 ; 3$ - по формуле (2-296) из [1] (третье приближение) 
Результаты расчётов температуры по формуле (17) для первого, второго и пятого приближений представлены на рис. 2, 3. Здесь же даны значения температур, полученных по методу [1] в третьем приближении. Из анализа результатов следует, что стабилизация температуры по длине канала происходит при $\eta \approx 0,25$. С уменьшением величины $\eta$ профиль температуры смещается в сторону невозмущенного потока (вблизи неподвижной пластины), что объясняется различными скоростями движения среды вблизи подвижной и неподвижной стенок. Ввиду того что конвективный теплоперенос в зоне больших скоростей течения среды оказывается бо́льшим, при малых значениях $\eta$ температура вблизи подвижной стенки оказывается меньшей. С увеличением $\eta$ происходит стабилизация теплообмена и при $\eta>0,25$ профиль температуры становится практически симметричным относительно центра канала $(\xi=0,5)$ (см. рис. 2, a).

На рис. 4 дано изменение невязки уравнения (13) для второго и пятого приближений. Анализ полученных результатов приводит к заключению о том, что при $\eta>0,01$ невязка уравнения (13) в пятом приближении практически равна нулю.

\section{Выводы.}

1. На основе ортогонального метода Л. В. Канторовича получено приближенное аналитическое решение нелинейной задачи теплообмена в жидкости (течение Куэтта) с учётом теплоты трения при граничном условии третьего рода на движущейся стенке, позволяющее выполнять исследование температурного состояния движущейся жидкости для малых значений продольной пространственной координаты.

2. Анализ полученных результатов приводит к заключению, согласно которому при малых значениях продольной координаты $(\eta<0,01)$ наблюдается смещение максимума температуры в сторону неподвижного потока. Следовательно, при малых (и сверхмалых) значениях $\eta$ температура на движущейся стенке значительно ниже, чем температура на границе возмущенного потока - на границе образующегося при движении тела пограничного слоя. Отсюда можно сделать вывод о том, что для защиты устройств, движущихся с высокой скоростью в различных средах, следует так организовать срыв пограничного слоя, чтобы не происходила стабилизация температурного поля в потоке, способствующая перегреву стенки от диссипации энергии. Полученное решение позволяет выполнить необходимые для этого расчёты при конкретных исходных данных задачи. 


\title{
БИБЛИОГРАФИЧЕСКИЙ СПИСОК
}

1. Цой П. В. Методы расчета отдельных задач тепломассопереноса. М.: Энергия, 1971. 383 c. [Tsoi P. V. Methods for calculating individual problems in heat and mass transfer. Moscow: Eneriya, 1971. 383 pp.]

2. Кудинов В.А., Карташов Э. М., Калашников В. В. Аналитические решения задач тепломассопереноса и термоупругости для многослойных конструкций. М.: Высш. шк., 2005. 430 c. [Kudinov V.A., Kartashov É. M., Kalashnikov V. V. Analytical solutions of problem of heat and mass transfer and thermoelasticity for multilayered structures. Moscow: Vyssh. Shk., 2005. 430 pp.]

3. Канторович Л. В., Крылов В. И. Приближенные методы высшего анализа. М.-Л.: Физматлит, 1962. 708 с. [Kantorovich L. V., Krylov V.I. Approximate methods of higher analysis. Moscow, Leningrad: Fizmatlit, 1962. 708 pp.]

4. Кудинов В.А., Кудинов И.В. Теплообмен при течении Куэтта с учётом диссипации энергии // Энергетика. Изв. высш. учеб. заведений и энерг. объединен. СНГ (Минск), 2011. № 2. C. 43-51. [Kudinov V. A., Kudinov I. V. Heat transfer in Couette flow with the energy dissipation// Energetika. Izv. Vyssh. Ucheb. Zavedeniy Energ. Obyedin., 2011. no. 2. Pp. 43-51].

MSC: 80A17, 80M25

\section{HEAT TRANSFER IN COUETTE FLOW CORRECTED FOR ENERGY DISSIPATION BY THE THIRD KIND BOUNDARY CONDITIONS}

\author{
A. V. Eremin ${ }^{1}$, N. M. Budylnikov ${ }^{1}$, I. V. Kudinov ${ }^{2}$ \\ 1 Samara State Technical University, \\ 244, Molodogvardeyskaya st., Samara, 443100, Russia. \\ 2 Samara State University of Architecture and Civil Engineering, \\ 194, Molodogvardeyskaya st., Samara, 443001, Russia. \\ E-mails: a.v.eremin@list.ru, budylnikov@mail.ru, igor_koudinov@mail.ru
}

The analytical solution of the non-linear heat transfer problem for laminar flow of liquid in plane-parallel channel (Coutte flow) corrected for energy dissipation by the third kind boundary conditions on the moving wall is obtained by the Kantorovich and the orthogonal Bubnov-Galerkin methods. The solution allows to estimate the temperature state of liquid for the small values of longitudinal coordinate, where the displacement of temperature profile to the immovable wall takes place as the investigations have shown.

Key words: Coutte flow, heat exchangein in a liquid, analytical solution, orthogonal methods, energy dissipation.

Original article submitted $28 / \mathrm{IV} / 2012$;

revision submitted 22/VIII/2012.

Anton V. Eremin, Postgraduate Student, Dept. of Theoretical Basis of Heat Engineering \& Flow Mechanics. Nikolay M. Budylnikov, Postgraduate Student, Dept. of Theoretical Basis of Heat Engineering \& Flow Mechanics. Igor V. Kudinov (Ph. D. (Techn.)), Assistant, Dept. of Hydraulic and Heat Engineering. 